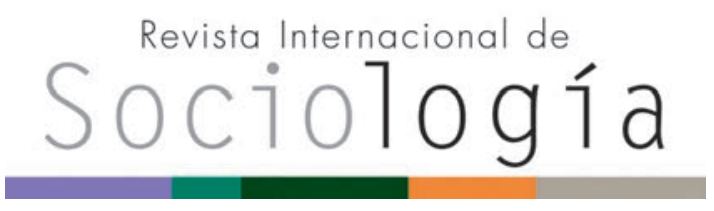

Revista Internacional de Sociología RIS

vol. 77 (2), e124, abril-junio, 2019, ISSN-L:0034-9712 https://doi.org/10.3989/ris.2019.77.2.17.139

\section{DESMONTANDO LA SEGURIDAD EN LAS POLÍTICAS DE EMPLEO: LA PROPUESTA EUROPEA DE FLEXIGURIDAD}

\author{
PAZ MARTíN \\ Universidad Complutense de Madrid \\ pazmartin@ucm.es \\ ORCID iD: https://orcid.org/0000-0002-2672-4424 \\ F. JosÉ TOVAR \\ Universidad Rey Juan Carlos \\ kiko.tovar.martinez@gmail.com \\ ORCID iD: https://orcid.org/0000-0003-4870-5432
}

Cómo citar este artículo / Citation: Martin. P. y F. J. Tovar. 2019. "Desmontando la seguridad en las políticas de empleo: la propuesta europea de flexiguridad". Revista Internacional de Sociología 77(2):e124. https:// doi.org/10.3989/ris.2019.77.2.17.139

\begin{abstract}
Resumen
A pesar de no tener muchos años de vida, el concepto de flexiguridad cuenta con una dilatada historia debido al peso sustantivo que se le ha concedido en todas las políticas de empleo europeas auspiciadas por el Modelo Social Europeo. Se trata de un concepto que se gesta en un contexto de regulación política laxa (soft governance) en el que no existe una tradición de políticas sociales y de empleo específica, al ser un espacio institucional internacional donde podrían confluir diversas tradiciones de los Estados miembros. Teniendo en cuenta estos aspectos, este artículo se propone profundizar en el significado y carácter ideológico-político del concepto de flexiguridad. Para ello, en primer lugar, indagamos en su contexto de producción y en las teorías y paradigmas de regulación en los que se basa; en segundo lugar, se realiza un análisis del discurso de algunos de los textos de la Comisión Europea centrados en este concepto, evidenciando la carga semántica desigual de flexibilidad y seguridad en su seno y la transformación semántica que la seguridad experimenta. Esta evidencia descubre una nueva realidad del mundo del trabajo en la que trabajador y empresario parecen haber intercambiado sus roles: el trabajador tiene el deber moral de empoderarse mediante la adquisición de capacidades adaptativas (su situación laboral dependerá de haberlo hecho o no), mientras que el empresario deviene en un agente vulnerable ante los nuevos flujos económicos.
\end{abstract}

\section{Palabras Clave}

Flexibilidad; Individualización; Mercados de trabajo transicionales; Paradigma de la activación; Vulnerabilidad.

\section{DISMANTLING SECURITY IN EMPLOYMENT POLICIES: THE EUROPEAN FLEXICURITY APPROACH}

Copyright: (C) 2019 CSIC. Este es un artículo de acceso abierto distribuido bajo los términos de la licencia de uso y distribución Creative Commons Reconocimiento 4.0 Internacional (CC BY 4.0).

Recibido: 03/10/2017. Aceptado: 09/10/2018 Publicado online: 04/06/2019

\begin{abstract}
Although it is not very old, the concept of flexicurity has a long history due to the substantive weight it has been given in all European employment policies under the auspices of the European Social Model. This is a concept that is developed in a context of lax political regula-tion (soft governance) and where there is no tradition of specific social and employment policies, as it is an in-ternational institutional space where different traditions of the Member States could converge. Taking these as-pects into account, this article aims to delve deeper into the ideological-political meaning and character of the concept of flexicurity. To this end, firstly, we investigate its production context and the theories and regulatory paradigms on which it is based, secondly, an analysis of the discourse of some of the European Commission's texts focusing on this concept is carried out, highlight-ing the unequal semantic load of flexibility and security within it and the semantic transformation that "security" undergoes. This evidence reveals a new reality in the world of work, in which workers and employers seem to have exchanged roles: workers have a moral duty to empower themselves with adaptive skills (their employ-ment situation will depend on whether or not they have done so) while employers become
\end{abstract} vulnerable to new economic flows.

\section{KEYWORDS}

Activation paradigm; Flexibility; Individualization; Transitional labour markets; Vulnerability. 


\section{INTRODUCCIÓN: FLEXIGURIDAD, ¿LA RECETA PERFECTA?}

Las instituciones europeas, en su pretensión de establecer un modelo económico y social común, han venido desarrollando una serie de políticas comunes de empleo creando diferentes vías para ponerlas en marcha (Goetschy 1999). En las cumbres de Ámsterdam (1997) y Lisboa (2000) se fijaron un número de ambiciosos objetivos, a conseguir en diez años, que hicieran de la economía de la UE la más competitiva y dinámica del mundo, siendo capaz de compaginar el crecimiento económico con la mejora cuantitativa y cualitativa del empleo y todo ello acompañado de una fuerte cohesión social (Comisión Europea 2003). El concepto de flexiguridad se difunde a nivel europeo en el marco de estas iniciativas que se configuran a través de la Estrategia Europea para el Empleo y del Modelo Social Europeo. Se trata de un concepto ambiguo, complejo y polisémico que, sin embargo, se alza como referente o concepto-guía de las actuaciones de los Estados miembros en materia de empleo y protección social.

Las nociones de flexiguridad, empleabilidad, activación y modelo social europeo se establecen como nociones-guía, marcos de referencia de las políticas de empleo y marcos interpretativos de lo social (Serrano 2005) porque, además de contribuir a canalizar el debate político, ejercen su influencia normativa a través del sistema de gobernanza multinivel que constituye el Método Abierto de Coordinación. Esto quiere decir que, si bien el proceso de integración europea es aún un proceso caótico, complejo e inacabado, es también productor de normas (Lefresne 1999).

Curiosamente, estos conceptos se vienen gestando y haciendo populares en un clima de creciente flexibilización del mercado de trabajo y una tendencia a la (des)calificación de los programas de seguros sociales como pasivos. La flexibilidad y la actividad se instituyen como las principales metas y referentes de las políticas públicas y sociales en toda Europa; frente a ellas, la legislación laboral se considera excesivamente rígida y los programas de seguro desincentivadores. En este contexto, la concepción de seguridad que habitaba y daba entidad al marco regulador y asegurador del empleo y el desempleo fordista-keynesiano resulta obsoleta e inoperante.

Por otro lado (y a pesar de los buenos propósitos), la fragilización de las relaciones laborales viene siendo, en los últimos años, un hecho demostrable en las estadísticas nacionales y supranacionales europeas (Comisión Europea 2010), que indican la disminución de los contratos indefinidos, la proliferación de contratos no indefinidos o la pérdida de poder de los sindicatos (Vielle \& Walthery 2003). Dicha fragilización está fuertemente asociada a una individualización de las relaciones laborales, esto es, el trabajador queda desprovisto de un marco colecti- vo de protección tanto en la situación de desempleo (paradigma de la activación) como en la de empleo (individual bargaining).

Algunos autores han puesto de relieve que estas tendencias de exaltación de la activación y la individualización favorecen la psicologización política del trabajo (Crespo, Revilla y Serrano 2005; McLaughlin 2010; Serrano, Martín y Crespo 2013). Siguiendo los mismos parámetros teóricos, autores como Bauman (2000 y 2006), Alonso (1999, 2001 y 2007), Rifkin (1997) o Sennet (2006) vienen señalando una serie de características comunes de una nueva etapa que podríamos denominar capitalismo tardío, entre ellas, el incremento de la presencia y eficacia de los procesos vulnerabilizadores de la fuerza de trabajo, como el aumento de la asimetría en la relación entre empleador y empleado, la precariedad y la fragmentación, entre otros.

Por tanto, en este trabajo abordamos la propuesta de flexiguridad como promotora, portadora y legitimadora de una nueva forma de entender el trabajo y su regulación. Una noción revestida de un carácter ideal acorde con los cambios culturales y productivos de la era post-industrial (Serrano y Keune 2014), que pretende condensar las posibilidades de ser de la organización del trabajo en los estados de bienestar europeos, desde hace algunas décadas adentrados en una crónica situación de crisis y reforma (Burroni y Keune 2011).

Dada esta potencialidad de transformación política que representa el concepto de flexiguridad europeo en un contexto de vulnerabilización de los trabajadores, resulta de particular interés indagar el tipo de equilibrio entre flexibilidad y seguridad que esta noción alberga y el significado de la noción de seguridad en su seno. En pos de esta indagación, en este trabajo, en primer lugar, nos aproximamos al contexto de emergencia del concepto de flexiguridad en el ámbito europeo y a alguno de sus referentes teóricos, como el paradigma de la activación y las teorías sobre los mercados de trabajo transicionales (entre otras) y prácticos, como la flexiguridad danesa, señalada como ejemplo de buenas prácticas en los documentos de la Comisión. Con ello pretendemos destacar la importancia de localizar los conceptos en su contexto a la hora de determinar su alcance práctico y significado y, por tanto, destacamos también la particularidad de la producción de políticas sociales europeas, tan vinculada a los procesos de justificación experta. Es fundamental considerar la especificidad y la historicidad de las categorías de análisis desde su contexto de producción (Maurice 1998). Así, tratamos de llegar a conocer el tipo de equilibrios o desequilibrios institucionales y las relaciones de fuerza que marcan, en su base, la propuesta política de la flexiguridad. En segundo lugar, analizamos la propuesta política de la flexiguridad en sí misma, aplicando el análisis crítico del discur- 
so a los primeros textos europeos que la integran y desarrollan con mayor amplitud: las Directrices integradas para el crecimiento y el empleo (2008-2010) (11.12.07) y la Comunicación de la Comisión para la propuesta de unos principios comunes de la flexiguridad (27.06.07). Tratamos de entender las relaciones de fuerza y los espacios semánticos que configuran estos textos y que, por tanto, remiten a un determinado tipo de equilibrio entre seguridad y flexibilidad. A la hora de aplicar este análisis, seguimos a autores como Foucault (1992), Bourdieu (2001) o Wodak y Meyer (2003), entre otros.

Finalmente, esbozamos unas conclusiones que resumen los principales hallazgos sobre la función reguladora de las políticas socio-laborales que emana de estos texto, en las cuales se aboga por favorecer la emergencia de alternativas al concepto y recuperar el significado genuino de seguridad en el ámbito de las políticas laborales.

\section{LA FLEXIGURIDAD EUROPEA Y SUS CON- TORNOS: CUESTIONES PARA UN DEBATE}

Los orígenes del término flexiguridad son, aún a día de hoy, discutidos. La primera publicación académica sobre flexiguridad aparece a finales de los años 90 (la de T. Wilthagen en 1998). En 1999, P. K. Madsen, economista danés, lo utiliza en una publicación para la OIT (Denmark: Flexibility, security and labour market success), refiriéndose a Dinamarca, cuyo caso siempre se ha considerado como el ejemplo ideal de aplicación, a pesar de las evidencias que cuestionan su transferibilidad a otros contextos (Mouriki 2009). Después, en el ámbito político danés, se considera que fue a partir de su mención por Rasmussen, líder del Partido Socialista danés, en un mitin en 2004, cuando la noción de flexiguridad comienza a ser más conocida popularmente (Bredgaard, Larsen y Madsen 2008).

A pesar de la diversidad de estudios y reflexiones en torno a la flexiguridad, podríamos concretar tres formas de aproximación al concepto: como estrategia política, como situación particular del mercado de trabajo y como concepto analítico (Bredgaard, Larsen y Madsen 2008).

En tanto que estrategia política, la flexiguridad ha sido ensalzada como una herramienta eficaz para combatir la segmentación del mercado de trabajo. Desde este punto de vista, consiste en la puesta en marcha de una serie de medidas que reducirían la distancia entre outsiders e insiders, A este respecto, Gallie (2007) ha elaborado una clasificación según la cual existen países con sistemas inclusivos, con sistemas dualistas y con sistemas de mercado. Los sistemas inclusivos son aquéllos con políticas tendentes a maximizar y ampliar los derechos de empleo a través de la fuerza de trabajo. En segundo lugar, encontramos los sistemas dualistas, en los que las políticas de empleo se balancean entre políticas de corte bajo para el empleo en general y políticas de protección fuertes para aquellos trabajadores en puestos considerados clave. Por último, los sistemas del mercado apuestan por un mínimo de protección de los empleados. Siguiendo a Heyes (2013) la flexiguridad es aplicada de dos maneras distintas según el tipo de sistemas que acabamos de describir. En el caso de los sistemas de mercado, se apuesta por una flexiguridad centrada en la segmentación externa (la que facilita las transiciones de los trabajadores en el mercado de trabajo), dando a los empleadores libertad para el despido. Por otro lado, en el caso de los sistemas inclusivos y dualistas se apuesta, sobre todo de la mano de los sindicatos, por una fuerte protección hacia el empleo para que los empresarios desarrollen una flexiguridad centrada en la segmentación interna como forma de controlar los costes laborales.

Este mismo autor nos presenta cuál ha sido la aplicación de la flexiguridad en cuatro países de la Unión Europea en el contexto de la crisis económica que se inicia en 2008, mostrando las divergencias de aplicación en función de las características particulares de cada uno (Heyes 2013). En el caso de Irlanda, ejemplo de país con un sistema político híbrido entre los sistemas descritos anteriormente, la flexiguridad se ha aplicado en mucha mayor medida en lo que hemos denominado el sector externo, a través de inversiones muy fuertes en formación continua (life long learning) para la población desempleada ${ }^{1}$, pero con medidas mínimas respecto a la prevención de pérdida de trabajo y apoyo a los trabajadores en situaciones precarias. En esa línea, la política de flexiguridad en el Reino Unido, en el contexto de la crisis, se ha centrado más en las personas sin empleo que en poner freno a la pérdida de empleo. En concreto, las medidas han ido destinadas a los jóvenes entre 18 y 24 años, incentivando su formación y su incorporación al mundo del trabajo en puestos séniores, a través de incentivos a las empresas. De otro lado, el sistema de protección social ha sufrido un adelgazamiento a través del aumento de la condicionalidad para el acceso a bajas laborales e incapacidades; o mediante la pérdida de derechos para aquellos desempleados que no acepten el primer empleo que se les ofrezca. Se trata de una política de empleo que entiende que una fuerte protección social limita y pone barreras a la creación de empleo y a la competitividad del país. En países como España, que ha fraguado un sistema laboral dualista en un contexto de fuerte desequilibrio de fuerzas y debilitamiento progresivo de los agentes sociales (Häusermann y Schwander 2010; Artiaga, Martín y Tovar 2014; Fernández 2014).

La noción de flexiguridad integraría diferentes tipos de seguridad y flexibilidad que se combinarían de formas distintas en función de las características del mercado de trabajo y los sistemas de protección de 
cada Estado. Desde este punto de vista, la flexiguridad constituiría más bien una herramienta capaz de baremar y, por tanto, evaluar tipos y grados de flexiguridad. La conocida matriz de Wilthagen es emblemática de esta aproximación (Wilthagen y Tros 2004).

Desde el propio nacimiento de la Europa social (Compromiso de Luxemburgo de 1997) se viene haciendo referencia a las presuntas bondades de aunar flexibilidad del mercado de trabajo con seguridad para los colectivos más desfavorecidos. Así, por ejemplo, aparece en el Libro Verde de la Comisión de 1997: Partenariado para una nueva organización del Trabajo la necesidad de compaginar flexibilidad y seguridad. Posteriormente, la palabra 'flexiguridad' se hace frecuente en los textos de las líneas directrices de 2005-2008. Es en enero de 2006 cuando, por primera vez, se hace una referencia más exclusiva y explícita al término en el Consejo Ministerial informal celebrado en Austria, quedando constancia de ello en el discurso del Comisario de Empleo, Asuntos Sociales e Igualdad de Oportunidades. En marzo de este mismo año, la Presidencia anima a la reforma del mercado de trabajo y las políticas sociales desde la perspectiva integrada de la flexiguridad y al establecimiento de un acuerdo sobre principios comunes. Desde este momento, se suceden distintas conferencias centradas en el debate sobre este concepto. En ellas, la participación del Grupo de Expertos sobre Flexiguridad, constituido al efecto de acordar estos principios comunes y conducir a la UE en estos términos, está siempre presentes y es crucial. Finalmente, tras la Comunicación oficial de la Comisión sobre Flexiguridad, en junio de 2007, se aprueban los principios comunes sobre flexiguridad en diciembre del mismo año.

Las nociones de empleo y desempleo, seguridad y flexibilidad, exclusión e inserción, etc. adquieren significado y activan marcos de pensamiento distintos en función de los procesos históricos a partir de los cuáles han surgido y han sufrido desplazamientos de sentido. Aspectos culturales, relaciones de fuerza entre distintos actores y circunstancias materiales componen este contexto y estos procesos históricos. En definitiva, podríamos entender las políticas de empleo como discursos de verdad que son resultado de disputas insertas en procesos histórico-culturales concretos, que expresan relaciones de poder, distribuyen responsabilidades y producen sujetos (Martín 2013). Partiendo de este enfoque analítico, podríamos decir que la UE se instituye, a través del Método Abierto de Coordinación (MAC), como instancia de regulación laxa del empleo desde un vacío contextual (histórico y cultural) y hacia una diversidad de situaciones, historias y menciones relativas a la regulación del empleo, es decir, hacia una pluralidad de mundos del trabajo (Martín 2013).

Hablamos de un vacío histórico (en el sentido de historia extensa) y cultural porque no existe una his- toria de lo social común europea y lo cultural, a este respecto, se ha fraguado de manera particular en cada país. Sin embargo, la UE no es un espacio vacío ideológicamente o libre de cargas políticas, muy al contrario, es terreno de batallas de ideas (Serrano 2006) y búsqueda de distintas fuentes de legitimación para hacerlas valer, a través de la constitución de comités de expertos (comitología), en el espacio más amplio de la gobernanza global.

La creación de un proyecto social o de intervención en lo social en Europa comenzó siendo un objetivo más que secundario en las primeras evoluciones de la Comunidad Económica Europea. En la segunda mitad de los años noventa, siguiendo el impulso del Tratado de Maastricht, se produce una ampliación de las competencias sociales de las instituciones europeas y se establece el MAC. La institucionalización de la Estrategia Europea para el Empleo es el resultado de este proceso, que más tarde se extiende a otros dominios sociales como la inclusión social (2000), las pensiones (2001), la educación y la formación (2002) (Goetschy 2005).

El Consejo Europeo de Lisboa (marzo de 2000) institucionaliza una regulación suave (soft governance) sobre el cumplimiento de las líneas directrices en los Estados miembros, el Método Abierto de Coordinación. En el seno de esta regulación suave es fundamental el papel que juegan las Ilamadas "comunidades epistémicas" (Verdun 1999; Jepsen y Serrano 2005) lanzando nuevas propuestas, iniciativas y conceptos (Serrano 2006). En el caso de la flexiguridad europea, éstas se elaboran y reelaboran en los comités de expertos, se difunden a través del MAC y encuentran su base teórica en los paradigmas de la activación y las teorías de los mercados de trabajo transicionales. Son aproximaciones teóricas susceptibles de adquirir una orientación particular acorde con una determinada constelación de actores y relaciones de fuerza.

El paradigma de la activación implica una innovación en el ámbito de las políticas sociales, pues entraña más un mecanismo de intervención en y sobre el individuo que para el individuo. Serrano (2007) identifica en la base del paradigma de la activación tres factores fundamentales que marcan la diferencia con respecto al modelo anterior: una aproximación individualizada al problema, psicologicista, centrada en la modelación del comportamiento, de las actitudes y la motivación de los trabajadores; un énfasis en el empleo, esto es, en los aspectos económicos de la ciudadanía, antes que en los políticos y sociales, y un reforzamiento de la moral del contrato, en el sentido de contrato privado-liberal, asentada sobre los criterios de la reciprocidad y el mérito.

Serrano (2007) ha llegado a identificar dos vertientes axiológica y metodológicamente diferenciadas en el paradigma de la activación: la gestión terapéutica-moral y la gestión adaptadora de habilidades 
y cualificaciones (matching). La terapéutica-moral se guía, principalmente, por criterios de merecimiento y programas dedicados a la modelación de la personalidad, de modo que se torne en una 'personalidad' lo más activa posible, para que el individuo pueda auto-garantizarse la colocación o el mantenimiento del empleo y adecuada a las necesidades que demanda el trabajo. La gestión adaptadora de las habilidades, por su parte, mantiene una visión más relacional y social del individuo. Su preocupación no es tanto la disciplina moral como la adecuación y explotación de las capacidades y el potencial humano de los individuos según las demandas del mercado.

Las teorías de los mercados de trabajo transicionales comienzan a desarrollarse en Europa a partir de 1995. Su principal exponente es Günter Schmid, quien, junto a Gazier o M.J. Rodríguez, realiza trabajos en la misma línea que sirven a las propuestas institucionales internacionales, particularmente las de la UE, promoviendo y apoyando sus ideales de "movilidad protegida y trabajo decente". Se refieren a 'transiciones' como "trayectorias individuales", comprendidas en alguno de los siguientes tipos: de la educación al empleo; del trabajo doméstico al empleo; del desempleo al empleo y viceversa; de un empleo a otro; del empleo a la jubilación, otorgándole al individuo la ventaja de ciertos derechos dentro del mercado de trabajo, convirtiéndolas en "buenas transiciones" (Gazier 2008). Schmid (1995) realiza una aportación teórica fundamental en la transformación de la noción de lo que debe ser un mercado de trabajo que, si antes estaba compuesto de posiciones estables y duraderas, ahora se reviste de múltiples modalidades de transición, aparentemente, ajustadas a los ciclos de vida particulares.

Existe un consenso sobre los principios de gestión de estas transiciones (buenas transiciones):

1. Empoderamiento de los sujetos para armonizar ciclos de vida y trabajo

\section{Solidaridad}

3. Reparto de las responsabilidades: co-financiación

\section{Gestión descentralizada: coordinación}

Se realiza un ejercicio de naturalización y legitimación de las transiciones sobre el que se sustentan los discursos de la UE. Schmid (2008), establece las claves de una "empleabilidad avanzada" dentro de la flexiguridad cuando se refiere a la movilidad en el espacio, en el tiempo y entre distintos regímenes de trabajo; al mantenimiento de la relación de empleo o su restablecimiento lo antes posible, y al 'empoderamiento', que define como "el potencial para adaptarse' o 'la capacidad de los individuos para hacer frente al riesgo". La seguridad puede, entonces, convertirse en un instrumento de activación y la empleabilidad en una exigencia extensiva a toda la población y en todo momento de su vida, no sólo concerniente al desempleado. Estos paradigmas de intervención confirman el desplome de los pilares del sistema de empleo moderno, con su concepción de trabajo estable y permanente. Algunos autores establecen la crítica a este respecto en términos de re-mercantilización del trabajo (Gautié 2004; Serrano 2005; Palier 2002; Alonso 2007; Boltanski y Chiapello, 2002).

Pero también son muchos los autores que defienden esta visión dinámica de los Mercados de Trabajo Transicionales (MTT), proponiendo una reinstitucionalización flexible de los mercados clásicos de trabajo que, de alguna manera, amortiguaría las incertidumbres de las sociedades actuales, generando espacios de confianza a través de transiciones, negociaciones y reglamentaciones de las que participan los distintos actores implicados (Schömann et al. 2000). Los MTT tendrían en la flexiguridad su complemento normativo, formando un tándem en la visión de la política social y económica, posibilitando un equilibrio de intereses de todas las partes en busca del beneficio común a través de herramientas válidas para la resolución de los conflictos. Schmid y Schömann (2006) muestran cómo la intención final de las políticas de flexiguridad es dotarse de nuevos mecanismos institucionales que permitan la mejora de la empleabilidad y la flexibilidad de la población activa, posibilitando que "las transiciones compensen". Los MTT representarían la última propuesta europea para afrontar los desafíos e incertidumbres de la sociedad postindustrial, entendiéndose como el último mecanismo institucional dentro de la gestión de los riesgos sociales (Pérez del Prado, 2009).

Las instituciones europeas inician una dinámica de constante apropiación e importación, redefinición y exportación de términos que vierten sobre las agendas de los Estados miembros. Así ha venido ocurriendo, por ejemplo, con 'activación' y 'empleabilidad', antes que con 'flexiguridad'. En este sentido, algunos autores llegan a puntualizar que "la naturaleza reguladora de la UE radica en su capacidad de armonización de ideas, representaciones y metas políticas" (Palier 2002), detectando "un incremento de la influencia sociocognitiva de los discursos de las instituciones europeas" (Crespo, Revilla y Serrano 2005). No obstante, esta influencia no se hace sentir con la misma fuerza en todos los Estados miembros ni tiene las mismas consecuencias para cada uno de ellos, como ya hemos visto que señala Heyes (2013). De hecho, las instituciones europeas insisten en que no existe un único modelo de flexiguridad, sino que varia según el contexto institucional, social y económico de los países miembros. Sin embargo, en este marco de difusión y ensalzamiento del concepto, las instituciones europeas señalan el sistema de protección social y mercado de trabajo danés como el mejor ejemplo (Comisión europea 2003; 2007a; 2010). Las referencias al "triángulo de oro danés" como virtuosa combinación de contratos laborales flexibles, generosas prestaciones por desempleo y eficaces 
políticas activas del mercado de trabajo, han sido numerosas. Pero es interesante observar cómo, en el momento en que se ensalzan las características del modelo danés, este empieza a acusar desequilibrios entre estos tres pilares en favor de una versión más coactiva y workfarista de políticas activas ${ }^{2}$.

La mayoría de los autores señalan el inicio del deterioro de los programas de políticas activas y el recorte de los presupuestos sociales en 2001, cuando la derecha danesa llega al poder (Jorgensen 2009, Larsen y Andersen 2009), indicando además que, en 2007 , el proceso de desmantelamiento de la flexiguridad danesa se intensifica con la descentralización de los servicios de empleo, pasando de regionales a locales. Esto supone un debilitamiento del poder de los sindicatos, en términos de su capacidad de intervención, ya que su foro de participación en la configuración de las políticas de empleo se localiza en el nivel regional. Algunos autores han calificado este proceso de "descentralización centralizada", ya que la gestión local supuso, paradójicamente, mayores cotas de poder para el gobierno central sobre asuntos que antes estaban en manos de las organizaciones sindicales (Larsen 2009). Así, se institucionaliza el antagonismo entre las políticas activas y las prestaciones por desempleo, en términos de oposición entre lo activo (positivo) y lo pasivo (negativo).

Teniendo en cuenta la particularidad de la gobernanza social europea detallada en esta sección, es decir, la ausencia de tradición socio-histórica e institucional de lo social a nivel de la UE, la naturaleza ideológica de la gobernanza a través del MAC, el protagonismo de las comisiones de expertos, de las relaciones de fuerza entre actores en el seno de las instituciones europeas, así como de los países miembros con mayor influencia dentro de ellas o que se establecen como referentes (Dinamarca, en este caso), se alzan las cuestiones acerca de los aspectos a los que los textos fundacionales europeos sobre flexiguridad conceden más atención, sobre qué clase de (des)equilibrios entre flexibilidad y seguridad promueven estos textos.

Acontinuación, tratamos de responder a estas cuestiones profundizando en el análisis de la flexiguridad europea según se plantea en los principios comunes del 27 de junio de 2007 y en las Directrices para el crecimiento y el empleo (2008-2010). Específicamente, indagamos el significado de seguridad, por ser el elemento en disputa en un ambiente laboral pautado por conseguir la mayor flexibilidad posible. Una de las principales características de estos textos es su carácter especialmente retórico, hasta cierto punto abstracto y, al mismo tiempo, propagandístico, con metáforas y argumentaciones legitimadoras que inciden en el surgimiento efectivo de una realidad diferente e irrevocable, razón por la cual son especialmente significativos en términos de análisis normativo.

\section{EL IDEAL FLEXIGURO DE EMPLEO. RIES- GO, MOVIMIENTO E INCERTIDUMBRE: LAS NUEVAS REGLAS DEL JUEGO}

El objetivo de esta sección es ahondar en el sentido de las transformaciones que se promueven desde la propuesta europea de flexiguridad. Concretamente, se explora la relación entre empleo y desempleo y seguridad y protección social ${ }^{3}$, entendida como gestión de los riesgos sociales.

El análisis de los documentos Comunicación de la Comisión para la propuesta de unos principios comunes de la flexiguridad (COM(2007) 359final) y las Directrices integradas para el crecimiento y el empleo (2008-2010) (COM(2007) 803final) se estructura en cuatro aspectos centrales para la estrategia política que describen y promueven. Nos referimos a: a) descripción y definición del contexto, metas y objetivos de la flexiguridad, desde los que ésta se legitima y se hace incluso necesaria; b) descripción y atribuciones de la noción de 'seguridad' y sus nuevos componentes cognitivo-normativos (flexibilidad y seguridad son intercambiables en el marco de la flexiguridad); c) representación de la relación entre trabajador y empresario, y d) términos y naturaleza de la relación (contrato) entre Estado y ciudadano. La construcción de un sentido de seguridad en relación con el empleo y el desempleo está ligada a los valores y elementos cognitivos que dan contenido a estos aspectos.

Una definición de la realidad como "economíasociedad del conocimiento" en la "era de la globalización" es el escenario en que estos factores se enclavan y al que seguidamente nos referimos. En este escenario, empresario y trabajador se presentan sometidos a las mismas presiones, en lugar de estar implicados en relaciones de poder asimétricas; la seguridad ya no es estabilidad y permanencia sino movimiento y esfuerzo personal y, por tanto, desde el Estado, el trabajador debe ser más bien incentivado y vigilado que protegido.

\section{a) Flexiguridad para un mundo cambiante $y$ globalizado}

Nos dedicaremos, en primer lugar, al análisis de aquellos fragmentos que dibujan un panorama desde el que los principios sobre flexiguridad y otros postulados complementarios a éstos adquieren sentido. Estos fragmentos perfilan un contexto mundial marcado por la internacionalización de la economía y que, en ocasiones, adquieren un tono de apremiante (y amenazante) necesidad de cambio. En ellos queda contenido el "principio de justificación por referencia a un bien común" (Boltanski y Chiapello 2002) o "la base de información de los juicios" (Sen 1987) de la estrategia de flexiguridad. 
Reflejan un discurso de "versión única" (GarcíaBorés 1996), monológico y homogeneizador de las circunstancias de cada Estado, tratando de transmitir una imagen del mundo incontestable. Su contenido está marcado por el uso de recursos propios de discursos totalizadores: impersonalización, pasivación, naturalización, etc.; tiene un cariz eminentemente prescriptivo y con carácter de urgencia.

"Sin embargo, Europa no se está ajustando tan bien como podría a los shocks que le son impuestos desde su economía. Esto puede que agrave las preocupaciones relativas a subcontratación y relocalización e incremente las desigualdades y distancias entre trabajadores cualificados y no cualificados" (Comisión Europea 2007b: 3)

En este fragmento, Europa se presenta personificada como un agente abstracto y sufridor de impactos, de los que se deriva un "puede que agrave" con tintes a medio camino entre la amenaza y la incertidumbre. Resulta un fragmento propio de un discurso vacío y retórico, en el que se mencionan situaciones concretas (subcontratación, relocalizaciones, desigualdades), pero no causas claras de las mismas (la falta de ajuste europeo a los "shocks" de la economía).

Desde la UE, la lógica del capitalismo flexible se ha categorizado como la lógica de "una economía basada en el conocimiento", que nos informa de (construye) las características de la experiencia colectiva actual en las democracias europeas y de su línea de explotación más ventajosa.

\footnotetext{
"La UE y sus Estados Miembros necesitan progresar más hacia una dinámica y exitosa economía del conocimiento, esparciendo más incluso los beneficios de la prosperidad a través de la sociedad. Deben resultar más ganadores ${ }^{4}$ de los procesos de cambio y mayor movilidad ascendente. Más 'no tener' tiene que transformarse en 'tener'" (Comisión Europea 2007b: 3).
}

"Esparcir los beneficios de la prosperidad" es, de nuevo, una prueba de la retórica que inunda el texto y que, en este caso, desplaza términos más directos, de tono más pragmático y que se encontraban en el centro del debate sobre el bienestar en momentos anteriores, como 'redistribución'. El lenguaje típico de los discursos sociales de la era keynesiana (con términos como 'justicia social' y 'redistribución') queda sepultado por el de la competición: "más ganadores", "mayor movilidad ascendente". Éstas son las aportaciones que se esperan de una economía del conocimiento mencionada en positivo, siendo introducida por los calificativos "dinámica y exitosa".

Se realiza una descripción del cambio drástica y explícita al mencionar el objeto de oposición: las "industrias tradicionales". Esta diferenciación radical antes-ahora permite realizar apología y legitimación de la formación continua y de la producción de capital humano. Así, "actualizarse constantemente" es condición de adaptación en la era de la "innovación y el cambio tecnológico".

\section{"Economías basadas en el conocimiento y basadas en los servicios requieren diferentes habilidades de las que precisaban las industrias tradicionales; ha- bilidades que además necesitan actualizarse cons- tantemente para hacer frente a la innovación y el cambio tecnológico" (Comisión Europea 2007b: 31).}

Existen más ejemplos de cómo las descripciones de un contexto macro se deslizan hacia afirmaciones contundentes sobre las circunstancias de la vida cotidiana, marcada por los cambios rápidos y la complejidad que inunda la vida laboral.

"La manera en que los ciudadanos europeos viven y trabajan está cambiando rápidamente" (Comisión Europea 2007a: 3).

"La vida laboral está llegando a ser más compleja ya que los patrones de trabajo se diversifican, llegan a ser más irregulares y es preciso que un número incrementado de transiciones se gestionen exitosamente a través de los ciclos de vida" (Comisión Europea 2007a: 31).

Presentan así una globalización penetrante en los resquicios de la sociedad e implacable (totalitaria) en sus efectos (imposiciones) a todos los niveles. Las impersonalizaciones como "se diversifican" y el carácter prescriptivo de afirmaciones como "es preciso" dan cuenta de ello. La globalización responde a alguna clase de fuerza sobrenatural que sólo una estrategia de flexiguridad puede aprovechar de la manera más ventajosa para todos. Es homogeneizadora de problemas y de soluciones.

"Flexiguridad (...) tiene también como meta la ayuda a empleados así como a empleadores para que aprovechen en su totalidad las oportunidades que les presenta la globalización" (Comisión Europea 2007a: 4).

Flexiguridad y globalización constituyen un binomio que actúa como disolvente de oposiciones y distensionador de conflictos empleado-empleador. La flexiguridad encierra los tipos de modificación que hay que promover para adaptarse al cambio. Este discurso general de hermanamiento entre flexiguridad y globalización incluye también menciones a las últimas metas que se persiguen desde esta transformación, los últimos fines en función de los que actuar merece la pena.

"Una aproximación de flexiguridad integrada es fundamental para el sostenimiento del crecimiento económico y el reforzamiento de la cohesión social" (Comisión Europea 2007a: 26).

En este contexto eminentemente competitivo la seguridad no remite a un estadio estático; de hecho, seguridad se hace intercambiable con flexibilidad, resultando ser uno de los motores que impulsan ese cambiar para adaptarse al cambio. 


\section{b) Seguridad y flexibilidad: conceptos inter- cambiables}

En el centro del discurso de la flexiguridad resulta interesante cómo flexibilidad y seguridad no se describen como conceptos opuestos, que sólo es posible compaginar sacrificando partes de uno y otro $^{5}$. Por el contrario, se les dota de una complementariedad total. Los dos conceptos, esencialmente opuestos, albergan las mismas prescripciones de actuación y promueven el mismo tipo de cualidades en el ciudadano: adaptabilidad, disponibilidad, polivalencia, preferencia por el cambio, etc., conforme a las demandas del mercado. Detengámonos, en primer lugar, en la definición de seguridad que ofrece el texto de la propuesta de principios de la flexiguridad.

\begin{abstract}
"La seguridad (...) es más que sólo mantener el puesto de trabajo de uno: es equipar a la gente con las habilidades que les permitan progresar en sus vidas laborales, y ayudarles a encontrar nuevo empleo. Tiene que ver con prestaciones de desempleo adecuadas para facilitar las transiciones. Finalmente, abarca oportunidades de formación para todos los trabajadores, especialmente, trabajadores de cualificación baja y trabajadores mayores." (Comisión Europea 2007a: 5).
\end{abstract}

El que la seguridad sea "más que sólo mantener el puesto de trabajo de uno", "equipar a la gente con habilidades" desvela la potencial inseguridad que reside en la falta de habilidades. Esto implica un desplazamiento de la seguridad desde algo externo al sujeto, que no depende de él, hacia la seguridad como capacidad y bagaje de cada uno. Las habilidades de cada uno son su fuente particular de seguridad.

Esto nos informa de una intervención política más en la persona del trabajador que para su persona. En los textos analizados, cuando se habla de seguridad están ausentes las menciones de la garantía en el mantenimiento del puesto y la dotación de ayudas en caso de pérdida. La seguridad, tal y como aparece en este extracto, es definida no tanto como un derecho, sino como un logro personal.

Esta definición evoca más bien una dinámica de tránsito entre distintos empleos que unas circunstancias puntuales de pérdida del puesto de trabajo. La seguridad es, entonces, instrumento de activación. El protagonismo que las transiciones adquieren en el enfoque de la flexiguridad parece apuntar a una radicalización de la empleabilidad como principal mecanismo asegurador (o auto-asegurador). La empleabilidad ya no es una receta sólo indicada para comenzar o volver a trabajar, sino también para continuar haciéndolo.

La garantía de seguridad viene dada por el auto-aseguramiento. Es así que la técnica aseguradora en el proyecto de flexiguridad lleva consigo una fuerte apelación a la responsabilidad personal, frente a la técnica aseguradora del estado providencia (estado de Bienestar keynesiano), asentada sobre el principio de la solidari- dad colectiva. Las prestaciones por desempleo se ponen al servicio de las transiciones. Éstas ya no tienen como misión principal la garantía del sustento en caso de "pérdida de los medios de vida" sino la facilitación de las transiciones (progresar en sus vidas laborales).

\begin{abstract}
"Las prestaciones para los ciudadanos y la sociedad deberían acumularse desde un aumento de movilidad de los trabajadores entre empresas. Los trabajadores estarán más inclinados a correr riesgos asociados a las transiciones de empleo si los subsidios son adecuados durante los períodos de transición y si las posibilidades de nuevos trabajos son reales" (Comisión Europea 2007a: 14).
\end{abstract}

En este fragmento, un criterio del merecimiento de las prestaciones sociales aparece en consonancia con esta moral de la transición, ya que parecen quedar condicionadas a "un aumento de movilidad de los trabajadores entre empresas". En definitiva, se trata de hacer que los trabajadores estén más dispuestos a correr riesgos (a cambio de algo).

"Los individuos necesitan incrementadamente seguridad en el empleo, más que seguridad en el puesto de trabajo, dado que cada vez menos gente tiene el mismo trabajo para toda la vida" (Comisión

Europea 2007a: 3).

"Seguridad en el empleo más que seguridad en el puesto de trabajo" se constituye como el lema asegurador de la flexiguridad. Esta frase deconstruye, por completo, el significado original de la seguridad en el mundo del trabajo, pues promueve el aseguramiento del constante tránsito frente al mantenimiento del puesto (eso era algo seguro), significado anterior propio de la noción. En un mundo laboral siempre agitado, la seguridad no se puede asimilar a una buena ancla para casos de tormenta, sino a un buen remo. Hay que estar siempre dispuesto a navegar y contar con las habilidades necesarias, más adecuadas, para seguir de isla en isla (de trabajo en trabajo).

El resultado es una confusión-difusión de los conceptos de flexibilidad y seguridad, cuando antes se percibían sustancialmente opuestos.

\section{c) Disolución del antagonismo trabajador- empresario}

Esta intercambiabilidad de las nociones de flexibilidad y seguridad está íntimamente ligada a la ruptura con una representación dialéctica de la realidad laboral. En los textos analizados, empresario y trabajador son tripulantes del mismo barco, son objeto y sujeto de esfuerzos similares.

“Desarrollar (...) más sistemáticamente estrategias políticas para mejorar la adaptabilidad de los trabajadores y las empresas" (Comisión Europea 2007b: 4).

"La adaptación requiere un mercado de trabajo más flexible combinado con niveles de seguridad que se dirijan simultáneamente a las necesidades de los empleadores y los empleados" (Comisión Europea 2007a: 3). 
En estos fragmentos, el uso de los adverbios "sistemáticamente" y "simultáneamente" designando un modo ideal de actuación pública para hacer frente a las necesidades de empresarios y trabajadores es representativo de un fuerte grado de disolución de la oposición empresario- trabajador desde la perspectiva de las instituciones. Por otro lado, "adaptabilidad" y "adaptación" son recomendaciones dirigidas tanto a empresarios como a trabajadores, por lo que parecen indicar que tanto unos como otros enfrentan similares situaciones.

La noción de flexiguridad europea se presenta confusa o ambigua a este respecto. En ocasiones, desde Europa, se menciona la flexiguridad como promotora de nuevas formas de compensación (trade-off) mientras que, en otras, se la considera más bien una estrategia por la que "todos ganan" (win-win approach). A nivel interpretativo y conceptual, estas dos aproximaciones entrañan visiones contrapuestas de las relaciones laborales. Hablar de compensación implica, de hecho, el reconocimiento a priori de una situación de desventaja que necesita ser contrarrestada, partiendo de desiguales necesidades entre dos grupos; sin embargo, si hablamos de un "todos ganan", estamos asumiendo la existencia de medidas y acciones que son per se favorables a trabajadores y empresarios sin que haya necesariamente una intención compensatoria en ellas: "Flexicurity is claimed to create new winwin relations between former adversaries" (Bredgaard, Larsen y Madsen 2008: 308). Por otra parte, desde la flexiguridad europea se hace hincapié en los acuerdos individuales (individual agreements) entre trabajador y empresario, en beneficio de intereses particulares en cada caso y para favorecer los ciclos de vida personales. Por tanto, la versión de la flexiguridad europea supone la puesta en cuestión del carácter dialéctico de las relaciones laborales, contribuyendo a los procesos de individualización y descolectivización del trabajo (Fernández, Martín y Serrano 2012).

Esta disolución del antagonismo entre empresario y trabajador parece encontrarse en la base cognitiva de la (des)regulación jurídico-formal o exógena de la relación de trabajo, al acabar con la conciencia de un contratante débil. En contrapartida, se traduce en un incremento de la responsabilidad personal que encauza al trabajador hacia experiencias de autonomía e independencia paradójicamente ineludibles.

"La efectividad de las políticas activas de mercado de trabajo correlaciona positivamente con una legislación de protección del empleo menos estricta" (Comisión Europea 2007a: 7).

En definitiva, el trabajador ha de desarrollar las habilidades de exhibición de un rol para el que la protección es prescindible. El trabajador competente, en esta nueva configuración del bienestar, es un trabajador tan flexible como la forma de producción: disponible, creativo, comunicativo, autónomo. Se produce el salto cognitivo de la norma salarial del empleo a la norma empresarial del empleo (Prieto, 2010) que, sin lugar a dudas, contiene una definición diferente de trabajador. Pasamos de lo que tradicionalmente se ha llamado "el trabajador asalariado" a lo que algunos autores han denominado "el trabajador-empresario de sí mismo” (Crespo y Serrano 2002).

\section{d) Nuevos términos del contrato Estado-in- dividuo: nuevo equilibrio entre derechos y deberes}

La transformación de los términos del contrato Estado-individuo se produce conforme a una representación del ciudadano que ha de hacerse cargo de sí mismo. La preocupación consolidada por el aprovechamiento ilegítimo de las prestaciones públicas referido, sobre todo, a la idea de que el cobro de la prestación desincentiva la búsqueda de y la implicación en el empleo, da lugar a un endurecimiento de los criterios de merecimiento de las prestaciones. Todo este recelo hacia la dependencia del individuo respecto al Estado transforma las relaciones entre ambos (Serrano y Martín 2012). Éstas, al mismo tiempo que se hacen más personalizadas, precisan la puesta en marcha de toda una maquinaria de control que cuenta con un elemento material: las nuevas tecnologías de la información y la comunicación; y un elemento jurídico-formal: la invención de nuevas figuras o reglamentaciones que entrañan nuevos deberes para el solicitante de empleo, es decir, el reforzamiento de la condicionalidad para el acceso a las prestaciones. Se apela al reforzamiento del control y seguimiento en la aplicación de las políticas de empleo.

\section{“(...) revisión continua de los incentivos y frenos} que resultan de los impuestos y los sistemas de prestaciones, incluyendo la gestión y condicionalidad de las prestaciones..." (Comisión Europea 2007b: 30).

"Buenos sistemas de subsidio (...) puede que tengan un efecto negativo sobre la intensidad de las actividades de búsqueda del nuevo empleo" (Comisión Europea 2007b: 6).

Esta tendencia en el nuevo contrato entre Estado e individuo puede ser calificada de privatizante en, al menos, dos sentidos: tanto en el de adopción de una lógica de corte ideológico mercantilista y liberal basada en el incentivo, como en el de hacer de la relación entre Estado y ciudadano una relación más de tipo privado (entre particulares) que público (entre ciudadano y administraciones), como si se tratara de un contrato individual. Todo ello se manifiesta en los discursos a favor del reforzamiento de las condiciones de acceso y mantenimiento de la prestación y el llamamiento a un reparto de responsabilidades, relacionado con una fuerte apelación a los deberes del trabajador. Las referencias a la seguridad social están, en muchos casos, atravesadas por este principio derecho-y-deber, para alcanzar la meta de coste efectivo. Resulta interesante ver cómo, en el siguiente fragmento, la mejora de la seguridad social aparece asociada a un "gasto 
público adicional o reorganizado", al "seguimiento o condicionalidad" y todo ello tratando que el "coste" sea "efectivo". Resulta significativo cómo se relaciona un tipo de gestión política del desempleo con un tipo de gestión económica del desempleo.

\begin{abstract}
"Mejorar la seguridad social (...) puede que requiera un gasto público adicional o reorganizado que debe ir acompañado de un seguimiento y condicionalidad de las prestaciones para asegurar que tal gasto sea un coste efectivo" (Comisión Europea 2007a: 14).
\end{abstract}

Como consecuencia, el contrato social (Estadoindividuo) comienza a adoptar tintes de contrato privado, pero, en este caso, acusado de una fuerte asimetría y marcado por la jerarquía, en detrimento de los derechos del trabajador.

\section{A MOdO DE CONCLUSIÓN: HACER FRENTE A LA FLEXIGURIDAD COMO CONCEPTO FUNDADOR}

La producción de documentos oficiales sobre empleo y protección social por parte de la UE constituye una tentativa de regulación internacional de la cuestión social. Este tipo de producción política, carente de la legitimidad de los procedimientos democráticos estatales, se hace efectiva a través de la llamada gobernanza suave (soft governance).

La desequilibrada carga semántica de flexibilidad y seguridad en el concepto de flexiguridad, que no pocos autores han señalado en los últimos años, se pone de relieve en el presente trabajo: (i) a través de una aproximación a la propuesta europea de flexiguridad desde su anclaje social, es decir, de una aproximación a la especificidad e historicidad de esta categoría en su contexto de emergencia y (ii) a través del análisis crítico del discurso de algunos de los documentos europeos clave sobre flexiguridad.

Las conclusiones de estos análisis presentan la flexiguridad europea como una categoría resumen de un nuevo marco interpretativo y de intervención en relación con el tipo de (des)equilibrio entre protección social o justicia social y desarrollo económico. Difundida desde las instituciones europeas, la flexiguridad integra en un sólo discurso, compacto aunque no exento de ambigüedades, las reformas que el Estado Social y el mercado de trabajo sufren a partir de la década de los ochenta y trata de configurar, frente a la propuesta keynesiano-fordista, un nuevo modelo de gestión.

La UE extiende sus recomendaciones y líneas directrices desde una vacío histórico y cultural, en el sentido de que la UE no tiene una historia de lo social común, hacia una pluralidad de situaciones, historias y menciones de la regulación de lo social. En este contexto, las relaciones de fuerza entre actores, entre instancias institucionales y entre Estados miembros aglutinan todo el protagonismo que, en los ca- sos nacionales, además, se reparte/comparte con el peso de la historia y la tradición cultural e institucional de lo social. Por eso podemos decir que el peso de lo ideológico, avalado por lo teórico-científico, es mayor en este contexto de producción de políticas sociales y de empleo. Además, en este ámbito de gobierno la generación de conceptos espectacularizantes es una herramienta de regulación potente (empleabilidad, activación, emprendimiento, flexiguridad) y un objetivo de la creatividad social europea. En este espacio acultural y ahistórico de la UE estos conceptos tienen cabida sin cuestionamiento, sin que sean malsonantes, sin que resulten ajenos porque es el espacio de la ajenidad, de todos y de nadie: sin cultura, sin lengua, sin identidad fuerte compartida. De ahí el papel fundamental que juegan los procedimientos de difusión de estos conceptos a través de conferencias y medios de comunicación.

En este trabajo exploramos la posible fundación de una nueva concepción de seguridad que se alimenta del paradigma de la activación, según lo define Serrano (2007), y de la teoría de los mercados de trabajo transicionales en el seno de la propuesta europea de flexiguridad, tal y como es formulada en los primeros textos oficiales sobre el concepto.

La noción de activación llega a ser sinónimo sustituto de empleabilidad, que comenzó a difundirse por las instituciones europeas durante los años 90. Un ejercicio de deconstrucción la noción de empleabilidad (Serrano 2000) muestra que ésta ha cedido su patrimonio semántico a los conceptos de activación y flexiguridad (Martín 2009: 161). Los conceptos de empleabilidad, activación y flexiguridad comparten una interpretación de la prevención como prevención contra la inactividad, a través de la adquisición de competencias metodológicas referidas a la búsqueda efectiva de empleo y de la formación como formación para la actividad (adecuación a las necesidades de la empresa). Se trata de que todo el mundo participe lo más intensamente posible del sistema productivo, considerándose esto un síntoma de integración, independencia y autonomía y, lo contrario, una señal de inadaptación, exclusión, incapacidad o pereza.

Apelando a la flexiguridad se intenta construir una nueva realidad del mundo del trabajo, donde el trabajador ya no debe querer la estabilidad en su puesto, debe ser un trabajador empoderado y dinámico, mientras que el empresario se convierte en una víctima de los nuevos flujos de la economía y necesita todas las facilidades para poder seguir siendo competitivo. Sólo con un modelo de trabajador dinámico y activo se puede conseguir una economía competitiva (Martín 2009).

Desde los argumentos de legitimación de este concepto que acabamos de explorar se ponen de manifiesto cuáles son los hitos de su constitución, sus entrañas. Éstas serían algunas de las premisas subyacentes al concepto: 
- La protección del empleo se presenta como un obstáculo al crecimiento en el nuevo entorno económico y perjudicial para la reducción del desempleo (efecto desincentivador) y de la segmentación.

- La exaltación del presunto modelo de flexiguridad danés. El modelo danés no existe como tal, pues la situación del mercado de trabajo danés es una realidad dinámica sujeta a las circunstancias socio-políticas de cada momento y a las relaciones de fuerza entre actores. Además, como se indica en la sección segunda, justo en el momento en que las instituciones europeas lo ensalzan como ejemplo a seguir, éste comienza a perder sus virtudes de equilibrio entre flexibilidad laboral, activación y protección.

- Exaltación de la competitividad y los nuevos requisitos de la competitividad.

- El desplazamiento semántico-político de la protección por desempleo (seguro) a la lucha contra el paro (activación). Esta lucha se pretende acometer a través de la flexibilización de los contratos (un mercado fluido de trabajadores) y desde la insistencia en la política activa.

Combatir o plantear alternativas a esta seguridad activadora, a este énfasis en el individuo, el trabajo y las dinámicas contractuales individuales que pueblan la propuesta europea de flexiguridad, nos conduce a la articulación de marcos interpretativos que entrañen una recuperación de las identidades colectivas y un fuerte compromiso con la reivindicación de otros aspectos de la vida ajenos al trabajo asalariado, ya sea en el mercado formal o informal.

Por tanto, parece necesario recuperar y dignificar el significado claro y uniforme de numerosos con- ceptos que están siendo modificados con vistas a la consolidación de un determinado Modelo Social Europeo, sin perder de vista que los modelos teóricos o políticos difundidos a través de las organizaciones internacionales entrañan ideales que pueden desencadenar funcionamientos de muy distinto signo en cada entorno nacional particular. Por eso, estamos de acuerdo con Bredgaard, Larsen y Madsen (2008) en que la flexiguridad puede tener resultados positivos dependiendo de la combinación de sus dimensiones y en qué contexto se aplique; pero es perjudicial que se considere positiva per se.

\section{Agradecimientos}

Este artículo es el resultado de gran parte de nuestras inquietudes investigadoras en torno a las transformaciones del trabajo y sus diferentes formas de regulación. Así, el proyecto financiado por el Ministerio de Educación y Ciencia en suplan I+D+I (2004-2007) titulado Evaluación cualitativa de las políticas de activación: los límites de lo activo y lo pasivo, dirigido por Amparo Serrano Pascual y del cual formamos parte como miembros del equipo de investigación, supuso un marco de trabajo perfecto para las reflexiones aparecidas en este artículo. Por ello, queremos agradecer a Amparo Serrano Pascual y al resto del equipo las consideraciones, recomendaciones, críticas y sugerencias que fueron surgiendo en torno a la noción de flexiguridad y que permitieron la realización de este artículo, así como sus lecturas y comentarios sobre el mismo. Queremos también expresar nuestro agradecimiento a los investigadores y profesores de CARMA (Universidad de Aalborg) y, especialmente, a Henning Jorgensen, que nos dieron su tiempo y todos los recursos a su alcance para poder conocer y comprender la versión danesa de la flexiguridad.

\section{NOTAS}

1. El porcentaje de desempleo en Irlanda fue el que sufrió la mayor subida de los países de la UE, pasando del 4,6\% en 2007 al 11,9 \% en 2009 (Comisión Europea, 2010).

2. Según Mailand (2010), entre las coaliciones que se crearon a la hora de realizar la negociación de los Principios sobre flexiguridad (2007), Dinamarca ya se situaba en la llamada "coalición minimalista", a favor de la menor intervención gubernamental posible en el mercado de trabajo.

3. La relación entre protección social y empleo es una de las líneas temáticas que ocupan el centro de la discusión actual en el ámbito de los estudios del bienes- tar, junto a las desigualdades de género y la exclusión social, según han señalado Hicks y Esping-Andersen (2005). Nosotros añadiríamos, además de éstas, los estudios comparativos y la reforma del estado de bienestar en un contexto de globalización política, económica y cultural.

4. Señalamos con cursiva y negrita las expresiones más relevantes al respecto de lo que pretendemos ilustrar.

5. El modelo keynesiano-fordista se basa, en gran medida, sobre la buena marcha de la alianza entre capital y derechos sociales, entre libertad y seguridad, si bien bajo el reconocimiento de la naturaleza opuesta de los elementos de esa alianza. 


\section{REFERENCIAS BibLIOGRÁFICAS}

Alonso, L.E. 1999. Trabajo y ciudadanía. Estudios sobre la crisis de la sociedad salarial. Madrid: Trotta.

Alonso, L.E. 2001. Trabajo y posmodernidad: el empleo débil. Madrid: Fundamentos.

Alonso, L.E. 2007. La crisis de la ciudadanía laboral. Barcelona: Anthropos.

Artiaga Leiras, A., M.P. Martín Martín y F.J. Tovar Martínez. 2014. "La consolidación del marco de la activación y la flexiguridad en España (1997 - 2009)". Pp. 241-280 en El paradigma de la flexiguridad en las políticas de empleo españolas: un análisis cualitativo, coordinado por C. Fernández Rodríguez y A. Serrano Pascual. Madrid: CIS.

Bauman, Z. 2000. Trabajo, consumismo y nuevos pobres. Barcelona: Gedisa.

Bauman, Z. 2006. Modernidad líquida. Buenos Aires: Fondo de Cultura Económica.

Boltanski, L. y Chiapello, Ė. 2002., El nuevo espíritu del capitalismo, Madrid: Ediciones Akal,

Bourdieu, P. 2001. ¿Qué significa hablar? Madrid: Akal.

Bredgaard, T., F. Larsen y P.K. Madsen .2008. "Flexicurity: In pursuit of a moving target". European Journal of Social Security 10(4): 305-325. https://doi. org/10.1177/138826270801000401

Burroni, L. y M. Keune. 2011. "Flexicurity: a conceptual critique". European Journal of Industrial Relations 17(1): 75-91. DOI: 10.1177/0959680110393189

Comisión Europea. 2003. L'emploi, l'emploi, l'emploi. Brussels: Task -force pour l'emploi.

Comisión Europea. 2007a. Hacia los principios comunes de la flexiguridad: más y mejor empleo mediante la flexibilidad y la seguridad. Bruselas: Comisión Europea. Asuntos sociales y Empleo. COM(2007)359final.

Comisión Europea. 2007b. Directrices integradas para el crecimiento y el empleo (2008-2010). Bruselas: Comisión Europea. Asuntos sociales y Empleo. COM(2007)803final.

Comisión Europea. 2010. El empleo en Europa 2010. Madrid: Ministerio de Trabajo e Inmigración.

Crespo, E. y A. Serrano. 2002. "El discurso de la Unión Europea sobre la sociedad del conocimiento". Reis: Revista española de investigaciones sociológicas, 97: 189-210.

Crespo, E., J.C. Revilla y A. Serrano. 2005. "La psicologización política del trabajo»". Psicología social y problemas sociales 2 : 53-60.

Fernández, C.J. 2014. "La creación de las bases del sistema de regulación del desempleo". Pp. 179-219 en El paradigma de la flexiguridad en las políticas de empleo españolas: un análisis cualitativo. coordinado por C. Fernández Rodríguez y A. Serrano Pascual. Madrid: CIS.

Fernández, C.J., P. Martín y A. Serrano. 2012. "Flexicurity and the semantic transformation of the concept of security: the modernisation of the Spanish social protection". International Journal of Society Systems Science 2: 150-166.

Foucault, M. 1992. El orden del discurso. Buenos Aires: Tusquets Editores.

Gallie D. 2007. Employment Regimes and the Quality of Work. Oxford: Oxford University press.

García-Borés, J. 1996. "La desarticulación de discursos y la versión única como fenómeno e instrumento de po- der". P.p. 339-352 en Psicologías, discursos y poder, coordinado por A.J. Gordo y J.L. Linaza. Madrid: Visor.

Gazier, J. 2008. "Qué son los mercados transicionales". Documento para el Seminario de políticas de empleo y reformas del mercado de trabajo: experiencias europeas, 17 de noviembre de 2008, Buenos Aires.

Gautié, J. 2004. "Repensar la articulación entre mercado de trabajo y protección social en el postfordismo". Cuadernos de Relaciones Laborales 22(1):147-184.

Goetschy, J. 1999. "The European Employment Strategy: Genesis and Development". European Journal of Industrial Relations 5(2): 117-137. https://doi. org/10.1177/095968019952002

Goetschy, J. 2005. "The European Social Dialogue in Innovations and New Paradigms" Transfer 11(3): 409422. https://doi.org/10.1177/102425890501100315

Häusermann, S. and H. Schwander. 2010. "Varieties of dualization? Labor market segmentation and insider outsider divides across regimes". Paper prepared for the Conference The Dualisation of European Societies? January $14-16^{\text {th }}$, Green Templeton College, University of Oxford.

Heyes J. 2013. "Flexicurity in crisis: European labour market policies in a time of austerity". European Journal of Industrial Relations 19(1): 71-86. https://doi. org/10.1177/0959680112474749

Hicks A. y G. Esping-Andersen. 2005. "Comparative an Historial Studies of Public Policy and the Weltfare State." Pp. 509-525 en The Handbook of Political Sociology, editado por T. Janoski, R. Alford, A. Hicks y M.A. Swartz. Cambridge: Cambridge University Press.

Jepsen, M. y A. Serrano Pascual. 2005. The European Social Model: an exercise in deconstruction. Journal of European Social Policy, 15(3): 231-245. https://doi. org/10.1177/0958928705054087

Jorgensen, H. 2009. "From a Beautiful Swan to an Ugly Duckling: the Renewal of Danish Activation Policy since 2003". European Journal of Social Security, 1(4): $337-$ 368. https://doi.org/10.1177/138826270901100401

Larsen, C.A. and J.G. Andersen. 2009. "How New Economic Ideas Changed the Danish Welfare State. The case of Neo-liberal Ideas and highly Organised Social Democratic Interests". Governance. An International Journal of Policy, Administration and Institutions 22(2): 239-261. https://doi.org/10.1111/j.1468-0491.2009.01434.x

Larsen, F. 2009. "How new governance and operational reforms can transform employment policies towards work-first - Lessons from the implementation of employment policies in the Danish municipalities". Paper for the symposium at the University of Chicago: Welfare States in Transition: Social Policy Transformation in Organizational Practice, 15th May 2009, Chicago.

Lefresne, F. 1999. Employability in the heart of the European Employment Strategy. European Review of Labour and Research 5: 460-480.

Madsen, P.K. 1999. "Denmark: flexibility, security and labour market success". Employment and training papers 53. ILO.

Mailand, M. 2010. "The common European flexicurity principles: How a fragile consensus was reached". European Journal of Industrial Relations 16(3): 241-257. https:// doi.org/10.1177/0959680110375134

Martín, M.P. 2009. "Questioning the employment system: the European flexicurity approach". The Journal of Industrial Relations and Human Resources. 11(5): 95-114. 
Martín, M. P. 2013. "Reinvención del gobierno del desempleo: Francia y España ante el marco referencial de la flexiguridad". Tesis doctoral. Departamento de Psicología Social y Antropología Social. Facultad de Ciencias Políticas y Sociología. Universidad Complutense de Madrid.

Maurice, M. 1998. "Les paradoxes de l'analyse societale. Retrospective et propective". En Document Seminaire. L.E.S.T. 98/8. L'analyse societale revisitée.

McLaughlin, K. 2010. "Psychologisation and the construction of the political subject as vulnerable object". Annual Review of Critical Psychology 8: 63-79.

Mouriki, A. 2009. "Flexibility and security: an asymmetrical relationship?". Working Paper 09/74. Amsterdam Institute for Advanced Labour Studies, University of Amsterdam.

Palier, B. 2002. Gouverner la sécurité sociale. Paris: Puf.

Pérez del Prado, D. 2009. "Edad, riesgos sociales y mercados transicionales. Nuevas respuestas ante nuevos retos". Pp. 153-175, en Trabajadores Maduros. Un análisis multidisciplinar de la repercusión de la edad en el ámbito social, dirigido por J.R. Mercader Uguina. Valladolid: Lex Nova.

Prieto, C. 2010. "Flexicurity: a question of norms or of social regimes of employment?" Artículo presentado en la conferencia Alternatives to Flexicurity: New Concepts and Approaches, 6 y 7 de mayo, Madrid.

Rifkin, J. 1997. El fin del trabajo: Nuevas tecnologías contra puestos de trabajo. El nacimiento de una nueva era. Barcelona: Paidós.

Schmid, G. 1995. Is full employment still possible? Transitional labour markets as a new strategy of labour market policy. Economic and Industrial Democracy 16 (3): 429-456.

Schmid, G. 2008. Full employment in Europe - Managing labour market transitions and risks. Cheltenham/ Northampton: Edwad Elgar.

Schmid, G. y K. Schömann. 2006. "El concepto de mercados de trabajo transicionales y algunas conclusiones para la política de empleo: el estado de la cuestión". Pp. 1540 en Los mercados de trabajo transicionales: nuevos enfoques y políticas sobre los mercados de trabajo europeos, compilado por L. Toharia. Madrid: Ministerio de Trabajo e Inmigración.

Schömann, K., S. Flechtner, R. Mytzek e I. Schömann. 2000. "Moving towards employment insurance: unemployment insurance and employment protection in the OECD". Discussion Papers / Wissenschaftszentrum Berlin für Sozialforschung, Forschungsschwerpunkt Arbeitsmarkt und Beschäftigung, Abteilung Arbeitsmarktpolitik und Beschäftigung, 00-201. Berlin: Wissenschaftszentrum Berlin für Sozialforschung $\mathrm{gGmbH}$. https://nbn-resolving.org/urn:nbn:de:0168-ssoar-115717
Sen, A. 1987. Sobre ética y economía. Madrid, Alianza editorial.

Sennett, R. 2006. La cultura del nuevo capitalismo. Barcelona: Anagrama.

Serrano, A. 2000. "El concepto de empleabilidad en la estrategia europea de lucha contra el desempleo: una perspectiva crítica". Revista del Ministerio de Trabajo y Asuntos Sociales 21: 137-150.

Serrano, A. 2005. "Del desempleo como riesgo al desempleo como trampa: ¿qué distribución de las responsabilidades plantea el paradigma de la activación propuesto por las instituciones europeas?". Cuadernos de Relaciones Laborales 2: 219-246.

Serrano, A. 2006. "Supranational expertocracy and the policies of production of identities". Pp. 227-248 en The European- a new global player?, editado por M. Khun. New York: Peter Lang.

Serrano, A. 2007. "Activation Regimes in Europe: A clustering exercise". Pp. 275- 316 en Reshaping Welfare States and Activation Regimes in Europe, editado por A. Serrano y L. Magnusson. Brussels: Peter Lang.

Serrano Pascual, A. y P. Martín Martín. 2012. "Du discours européen de la sécurité à la rhétorique de la dépendance". Les politiques Sociales 3\&4: 11-26.

Serrano Pascual, A., P. Martín Martín y E. Crespo Suárez. 2013. "La regulación paradójica del trabajo y el gobierno de las voluntades". Pp.115-144 en Crisis y precariedad vital, editado por B. Tejerina, B. Cavia, S. Fortino y J.A. Calderón. Valencia: Tirant lo Blanch.

Serrano, A. y M. Keune. 2014. Deconstructing flexicurity and developing alternative approaches: towards new concepts and approaches for employment and Social Policy. London: Routledge.

Verdun, A. 1999. The role of the Delors Committee in the creation of EMU: an epistemic community? Journal of European Public Policy 6(2): 308-328, https://doi. org/10.1080/135017699343739

Vielle, P. y P. Walthery. 2003. Flexibility and social protection. Dublin: European Foundation for the Improvement of Living and Working Conditions.

Wilthagen, T. 1998. "Flexicurity - A new paradigm for labour market policy reform?". Discussion Paper FSI, 98-202, Berlin: WZB.

Wilthagen, T. y F. Tros. 2004. "The concept of "flexicurity": A new approach to regulating employment and labour market". TRANSFER 10(2): 166-186. https://doi. org/10.1177/102425890401000204

Wodak, R. y M. Meyer. 2003. Métodos de análisis crítico de discurso. Barcelona: Gedisa.

PAZ MARTín es investigadora en el Departamento de Psicología Social de la Universidad Complutense de Madrid. Sus principales ámbitos de investigación son la comparación transnacional de políticas sociales, el análisis cualitativo de políticas públicas, la reforma de los estados de bienestar y la gobernanza social. Tiene más de 20 publicaciones sobre diversos temas, entre las más recientes (en co-autoría): "The reconstruction of resilience as a social and collective phenomenon. Poverty and coping capacity during the economic crisis" (European Societies) y "From 'Employability' to 'Entrepreneuriality' in Spain: youth in the spotlight in times of crisis" (Journal of Youth Studies).

FRANCISCO JOSÉ TOVAR es profesor ayudante doctor en el Departamento de Comunicación y Sociología en la Universidad Rey Juan Carlos. Sus principales líneas de investigación son el estudio crítico de las organizaciones, las políticas sociales y la identidad laboral y colectiva. Entre sus publicaciones más recientes destacan (en co-autoría): "La articulación de las entidades colectivas y sociales: una perspectiva situada" (Papeles del CEIC), "Collective identities in the age of restructuring: old and new class, space and communitiy based identities in six euroepan regions" (International Sociology) o "L'ideologie d l'autoresponsabilite totalisatrice: l'individu compétent et flexisûr" (Les politiques sociales). 